\title{
On the sufficiency of finite-frame LQR optimality conditions for 2-D Roesser models
}

\author{
Lorenzo Ntogramatzidis Michael Cantoni
}

\begin{abstract}
It is shown that the necessary optimality conditions, which arise from a variational analysis of the linear quadratic regulator (LQR) problem for two-dimensional (2D) Roesser models, are also sufficient. An expression for the optimal performance index is also provided.
\end{abstract}

\section{INTRODUCTION}

Two-dimensional (2-D) systems arise naturally in the modelling of processes where the variables are defined over space rather than time. The most widespread latent variable models for linear shift-invariant 2-D systems are the Roesser [1] and Fornasini-Marchesini [2] models. Optimal control problems for Fornasini-Marchesini models are addressed, over an infinite frame, in [3] and [4]. The optimal control of 2-D systems over a finite frame is studied in [5], within the context of Roesser models. There, a variational approach is used to obtain necessary conditions for optimality. In this paper, it is shown that the corresponding conditions are also sufficient for the linear quadratic regulator (LQR) problem. The solution of the equations which characterise the optimality condition is discussed and an expression for the optimal value of the performance index is provided.

Notation. Given a region $\mathcal{A}$ of $\mathbb{N} \times \mathbb{N}$ and a signal $s$ : $\mathbb{N} \times \mathbb{N} \longrightarrow \mathbb{R}^{n}$, we denote by $\left.s\right|_{\mathcal{A}}$ the restriction of $s$ to the region $\mathcal{A}$.

\section{Problem Statement}

Consider a linear shift-invariant Roesser model

$$
\left[\begin{array}{c}
h_{i+1, j} \\
v_{i, j+1}
\end{array}\right]=\left[\begin{array}{cc}
A_{1} & A_{2} \\
A_{3} & A_{4}
\end{array}\right]\left[\begin{array}{c}
h_{i, j} \\
v_{i, j}
\end{array}\right]+\left[\begin{array}{c}
B_{1} \\
B_{2}
\end{array}\right] u_{i, j}
$$

where, for all $i, j \in \mathbb{N}, h_{i, j} \in \mathbb{R}^{n_{h}}$ is the horizontal semistate, $v_{i, j} \in \mathbb{R}^{n_{v}}$ is the vertical semistate and $u_{i, j} \in \mathbb{R}^{m}$ is the control input. The matrices $A_{h}, h \in\{1, \ldots, 4\}$, and $B_{k}$, $k \in\{1,2\}$ are of suitable dimensions. By specifying the input $u_{i, j}$ over a bounded frame $\mathcal{Q} \triangleq[0, N] \times[0, M]$, and (south-west) boundary conditions

$$
\begin{aligned}
& h_{0, j}=a_{j} \in \mathbb{R}^{n_{h}}, \quad j \in[0, M], \\
& v_{i, 0}=b_{i} \in \mathbb{R}^{n_{v}}, \quad i \in[0, N]
\end{aligned}
$$

on the horizontal and vertical semistates, respectively, the model (1) produces a unique value for $h_{i, j}$ over $\mathcal{H} \triangleq[0, N+$

\footnotetext{
Lorenzo Ntogramatzidis and Michael Cantoni are with the Department of Electrical and Electronic Engineering, The University of Melbourne, Parkville VIC 3010, Australia $\{$ lnt, m. cantoni\}@ee.unimelb.edu. au
}

1] $\times[0, M]$ and $v_{i, j}$ over $\mathcal{V} \triangleq[0, N] \times[0, M+1]$. Let

$$
\begin{array}{r}
J\left(\left.h\right|_{\mathcal{H}},\left.v\right|_{\mathcal{V}},\left.u\right|_{\mathcal{Q}}\right) \triangleq \mathbf{h}_{N+1}^{\top} H \mathbf{h}_{N+1}+\mathbf{v}_{M+1}^{\top} V \mathbf{v}_{M+1} \\
+\sum_{(i, j) \in \mathcal{Q}}\left[\begin{array}{lll}
h_{i, j}^{\top} & v_{i, j}^{\top} & u_{i, j}^{\top}
\end{array}\right]\left[\begin{array}{ccc}
Q_{h} & Q_{h v} & S_{h} \\
Q_{h v}^{\top} & Q_{v} & S_{v} \\
S_{h}^{\top} & S_{v}^{\top} & R
\end{array}\right]\left[\begin{array}{l}
h_{i, j} \\
v_{i, j} \\
u_{i, j}
\end{array}\right],
\end{array}
$$

where $\mathbf{h}_{N+1} \triangleq\left[h_{N+1,0}^{\top} \ldots h_{N+1, M}^{\top}\right]^{\top}, \mathbf{v}_{M+1} \triangleq$ $\left[v_{0, M+1}^{\top} \ldots v_{N, M+1}^{\top}\right]^{\top}$ and the matrices $H=H^{\top} \geq 0$ and $V=V^{\top} \geq 0$ penalise the (north-east) boundary values of the horizontal and vertical semistates, respectively. It is assumed that with

$Q \triangleq\left[\begin{array}{cc}Q_{h} & Q_{h v} \\ Q_{h v}^{\top} & Q_{v}\end{array}\right], \quad S \triangleq\left[\begin{array}{c}S_{h} \\ S_{v}\end{array}\right], \quad \Pi \triangleq\left[\begin{array}{cc}Q & S \\ S^{\top} & R\end{array}\right]$

the matrix $\Pi$ is symmetric and positive semidefinite. Note that here it is not assumed that $R$ is non-singular. It is now possible to formally define the optimal control problem considered in this paper.

Problem 1: Find a control input $u_{i, j}$, for $(i, j) \in \mathcal{Q}$, the corresponding horizontal semistate $h_{i, j}$, for $(i, j) \in \mathcal{H}$ and vertical semistate $v_{i, j}$, for $(i, j) \in \mathcal{V}$, to minimize the quadratic performance index (3) under the constraints (1) and (2).

\section{MAin Results}

In [5], necessary optimality conditions for Problem 1 are obtained as a special case of a necessary optimality condition established therein for a non-linear version of the constraint (1), via a variational analysis of (3), augmented with the product of Lagrange multipliers and the constraints. The question of sufficiency remained unanswered in [5]. Below, it is established that the optimality condition is in fact also sufficient for the LQR Problem 1. The approach taken is similar in nature to that in [6], where the continuous 1-D problem is considered, and convexity is exploited to demonstrate sufficiency. Situations in which it is possible to express the equations that characterise the optimality condition more explicitly are also discussed.

Theorem 1: If $h_{i, j}, v_{i, j}$ and $u_{i, j}$ are optimal for Problem 1 , then $\lambda_{i, j} \in \mathbb{R}^{n_{h}},(i, j) \in \mathcal{H}$ and $\mu_{i, j} \in \mathbb{R}^{n_{v}},(i, j) \in \mathcal{V}$ exist such that $h_{i, j}, v_{i, j}, \lambda_{i, j}, \mu_{i, j}$ and $u_{i, j}$ satisfy the set of 
equations

$$
\begin{aligned}
\boldsymbol{\lambda}_{N+1} & =H \mathbf{h}_{N+1}, \\
\boldsymbol{\mu}_{M+1} & =V \mathbf{v}_{M+1}, \\
{\left[\begin{array}{l}
\lambda_{i, j} \\
\mu_{i, j}
\end{array}\right] } & =Q\left[\begin{array}{l}
h_{i, j} \\
v_{i, j}
\end{array}\right]+S u_{i, j}+A^{\top}\left[\begin{array}{l}
\lambda_{i+1, j} \\
\mu_{i, j+1}
\end{array}\right],(i, j) \in \mathcal{Q} \\
0 & =R u_{i, j}+S^{\top}\left[\begin{array}{l}
h_{i, j} \\
v_{i, j}
\end{array}\right]+B^{\top}\left[\begin{array}{l}
\lambda_{i+1, j} \\
\mu_{i, j+1}
\end{array}\right],(i, j) \in \mathcal{Q} \\
{\left[\begin{array}{c}
h_{i+1, j} \\
v_{i, j+1}
\end{array}\right] } & =A\left[\begin{array}{l}
h_{i, j} \\
v_{i, j}
\end{array}\right]+B u_{i, j},(i, j) \in \mathcal{Q} \\
\mathbf{h}_{0} & =a \\
\mathbf{v}_{0} & =b
\end{aligned}
$$

where we have defined $\boldsymbol{\lambda}_{N+1} \triangleq\left[\lambda_{N+1,0}^{\top} \ldots \lambda_{N+1, M}^{\top}\right]^{\top}$, $\boldsymbol{\mu}_{M+1} \triangleq\left[\mu_{0, M+1}^{\top} \ldots \mu_{N, M+1}^{\top}\right]^{\top}, \mathbf{h}_{0} \triangleq\left[h_{0,0}^{\top} \ldots h_{0, M}^{\top}\right]^{\top}$, $\mathbf{v}_{0} \triangleq\left[v_{0,0}^{\top} \ldots v_{N, 0}^{\top}\right]^{\top}, a \triangleq\left[a_{0}^{\top} \ldots a_{M}^{\top}\right]^{\top}$ and $b \triangleq$ $\left[\begin{array}{lll}b_{0}^{\top} & \ldots & b_{N}^{\top}\end{array}\right]^{\top}$. Conversely, if equations (4-10) admit solutions $h_{i, j}, v_{i, j}, \lambda_{i, j}, \mu_{i, j}$ and $u_{i, j}$, then the corresponding $h_{i, j}, v_{i, j}$ and $u_{i, j}$ minimize $J\left(\left.h\right|_{\mathcal{H}},\left.v\right|_{\mathcal{V}},\left.u\right|_{\mathcal{Q}}\right)$ subject to the constraints (1-2).

Proof: As aforementioned, the necessity of the conditions (4-10) for an optimum was established in [5]. Here we are interested in proving sufficiency. Let $h_{i, j}, v_{i, j}, u_{i, j}, \lambda_{i, j}, \mu_{i, j}$ be such that equations (4-10) hold. Let $\hat{h}_{i, j}, \hat{v}_{i, j}, \hat{u}_{i, j}$ be such that all the constraints of the optimal control problem are satisfied, i.e.

$$
\begin{aligned}
{\left[\begin{array}{l}
\hat{h}_{i+1, j} \\
\hat{v}_{i, j+1}
\end{array}\right] } & =A\left[\begin{array}{l}
\hat{h}_{i, j} \\
\hat{v}_{i, j}
\end{array}\right]+B \hat{u}_{i, j} \quad \forall(i, j) \in \mathcal{Q} \\
\hat{h}_{0, j} & =a_{j} \quad j \in[0, N], \quad \hat{v}_{i, 0}=b_{i} \quad i \in[0, M] .
\end{aligned}
$$

The aim here is to show that $J\left(\left.h\right|_{\mathcal{H}},\left.v\right|_{\mathcal{V}},\left.u\right|_{\mathcal{Q}}\right) \leq J\left(\left.\hat{h}\right|_{\mathcal{H}},\left.\hat{v}\right|_{\mathcal{V}},\left.\hat{u}\right|_{\mathcal{Q}}\right)$. By defining $p_{i, j} \triangleq\left[\begin{array}{lll}h_{i, j}^{\top} & v_{i, j}^{\top} & u_{i, j}^{\top}\end{array}\right]^{\top}, \quad \hat{p}_{i, j} \triangleq\left[\begin{array}{lll}\hat{h}_{i, j}^{\top} & \hat{v}_{i, j}^{\top} & \hat{u}_{i, j}^{\top}\end{array}\right]^{\top}$, $\hat{\mathbf{h}}_{N+1} \triangleq\left[\begin{array}{lll}\hat{h}_{N+1,0}^{\top} & \ldots & \hat{h}_{N+1, M}^{\top}\end{array}\right]^{\top} \quad$ and $\hat{\mathbf{v}}_{M+1} \triangleq\left[\begin{array}{lll}\hat{v}_{0, M+1}^{\top} & \cdots & \hat{v}_{N, M+1}^{\top}\end{array}\right]^{\top}$, one obtains

$$
\begin{aligned}
J\left(\left.\hat{h}\right|_{\mathcal{H}},\left.\hat{v}\right|_{\mathcal{V}},\left.\hat{u}\right|_{\mathcal{Q}}\right)- & J\left(\left.h\right|_{\mathcal{H}},\left.v\right|_{\mathcal{V}},\left.u\right|_{\mathcal{Q}}\right)=\hat{\mathbf{h}}_{N+1}^{\top} H \hat{\mathbf{h}}_{N+1} \\
& +\hat{\mathbf{v}}_{M+1}^{\top} V \hat{\mathbf{v}}_{M+1} \\
& -\mathbf{h}_{N+1}^{\top} H \mathbf{h}_{N+1}-\mathbf{v}_{M+1}^{\top} V \mathbf{v}_{M+1} \\
& +\sum_{(i, j) \in \mathcal{Q}}\left(\hat{p}_{i, j}^{\top} \Pi \hat{p}_{i, j}-p_{i, j}^{\top} \Pi p_{i, j}\right) .
\end{aligned}
$$

The positive semidefiniteness of the quadratic forms

$$
\left(\mathbf{h}_{N+1}-\hat{\mathbf{h}}_{N+1}\right)^{\top} H\left(\mathbf{h}_{N+1}-\hat{\mathbf{h}}_{N+1}\right)
$$

and

$$
\left(\mathbf{v}_{M+1}-\hat{\mathbf{v}}_{M+1}\right)^{\top} V\left(\mathbf{v}_{M+1}-\hat{\mathbf{v}}_{M+1}\right)
$$

implies that

$\hat{\mathbf{h}}_{N+1}^{\top} H \hat{\mathbf{h}}_{N+1}-\mathbf{h}_{N+1}^{\top} H \mathbf{h}_{N+1} \geq 2 \mathbf{h}_{N+1}^{\top} H\left(\hat{\mathbf{h}}_{N+1}-\mathbf{h}_{N+1}\right)$, $\hat{\mathbf{v}}_{M+1}^{\top} V \hat{\mathbf{v}}_{M+1}-\mathbf{v}_{M+1}^{\top} V \mathbf{v}_{M+1} \geq 2 \mathbf{v}_{M+1}^{\top} V\left(\hat{\mathbf{v}}_{M+1}-\mathbf{v}_{M+1}\right)$.
Likewise $\hat{p}_{i, j}^{\top} \Pi \hat{p}_{i, j}-p_{i, j}^{\top} \Pi p_{i, j} \geq 2 p_{i, j}^{\top} \Pi\left(\hat{p}_{i, j}-p_{i, j}\right)$ for all $(i, j) \in \mathcal{Q}$, so that

$$
\begin{aligned}
J\left(\left.\hat{h}\right|_{\mathcal{H}},\left.\hat{v}\right|_{\mathcal{V}},\left.\hat{u}\right|_{\mathcal{Q}}\right)- & J\left(\left.h\right|_{\mathcal{H}},\left.v\right|_{\mathcal{V}},\left.u\right|_{\mathcal{Q}}\right) \geq \\
& 2 \mathbf{h}_{N+1}^{\top} H\left(\hat{\mathbf{h}}_{N+1}-\mathbf{h}_{N+1}\right) \\
& +2 \mathbf{v}_{M+1}^{\top} V\left(\hat{\mathbf{v}}_{M+1}-\mathbf{v}_{M+1}\right) \\
& +\sum_{(i, j) \in \mathcal{Q}} 2 p_{i, j}^{\top} \Pi\left(\hat{p}_{i, j}-p_{i, j}\right) .
\end{aligned}
$$

By virtue of (6) it follows that for any $(i, j) \in \mathcal{Q}$,

$$
\Pi p_{i, j}=\left[\begin{array}{c}
\lambda_{i, j}-A_{1}^{\top} \lambda_{i+1, j}-A_{3}^{\top} \mu_{i, j+1} \\
\mu_{i, j}-A_{2}^{\top} \lambda_{i+1, j}-A_{4}^{\top} \mu_{i, j+1} \\
-B_{1}^{\top} \lambda_{i+1, j}-B_{2}^{\top} \mu_{i, j+1}
\end{array}\right]
$$

As such,

$$
\begin{aligned}
p_{i, j}^{\top} \Pi\left(\hat{p}_{i, j}-p_{i, j}\right)= & \lambda_{i+1, j}^{\top} h_{i+1, j}-\lambda_{i+1, j}^{\top} \hat{h}_{i+1, j} \\
& +\mu_{i, j+1}^{\top} v_{i, j+1}-\mu_{i, j+1}^{\top} \hat{v}_{i, j+1} \\
& +\lambda_{i, j}^{\top}\left(\hat{h}_{i, j}-h_{i, j}\right)+\mu_{i, j}^{\top}\left(\hat{v}_{i, j}-v_{i, j}\right)
\end{aligned}
$$

where equation (8) has been used. By (4) it follows that

$$
\begin{aligned}
\sum_{(i, j) \in \mathcal{Q}} \lambda_{i+1, j}^{\top} & \left(h_{i+1, j}-\hat{h}_{i+1, j}\right)-\lambda_{i, j}^{\top}\left(h_{i, j}-\hat{h}_{i, j}\right) \\
= & \sum_{j=0}^{M} \lambda_{N+1, j}^{\top}\left(h_{N+1, j}-\hat{h}_{N+1, j}\right) \\
& -\sum_{j=0}^{M} \lambda_{0, j}^{\top}\left(h_{0, j}-\hat{h}_{0, j}\right) \\
= & -\boldsymbol{\lambda}_{N+1}^{\top}\left(\hat{\mathbf{h}}_{N+1}-\mathbf{h}_{N+1}\right) \\
& -\sum_{j=0}^{M} \lambda_{0, j}^{\top}\left(h_{0, j}-\hat{h}_{0, j}\right) \\
= & -\mathbf{h}_{N+1}^{\top} H\left(\hat{\mathbf{h}}_{N+1}-\mathbf{h}_{N+1}\right) \\
& -\sum_{j=0}^{M} \lambda_{0, j}^{\top}\left(h_{0, j}-\hat{h}_{0, j}\right)
\end{aligned}
$$

and, similarly,

$$
\begin{aligned}
\sum_{(i, j) \in \mathcal{Q}} \mu_{i, j+1}^{\top} & \left(v_{i, j+1}-\hat{v}_{i, j+1}\right)-\mu_{i, j}^{\top}\left(v_{i, j}-\hat{v}_{i, j}\right) \\
= & -\mathbf{v}_{M+1}^{\top} V\left(\hat{\mathbf{v}}_{M+1}-\mathbf{v}_{M+1}\right) \\
& -\sum_{i=0}^{N} \mu_{i, 0}^{\top}\left(v_{i, 0}-\hat{v}_{i, 0}\right)
\end{aligned}
$$

so that

$$
\begin{aligned}
& J\left(\left.\hat{h}\right|_{\mathcal{H}},\left.\hat{v}\right|_{\mathcal{V}},\left.\hat{u}\right|_{\mathcal{Q}}\right)-J\left(\left.h\right|_{\mathcal{H}},\left.v\right|_{\mathcal{V}},\left.u\right|_{\mathcal{Q}}\right) \\
& \quad \geq-\sum_{j=0}^{M} \lambda_{0, j}^{\top}\left(h_{0, j}-\hat{h}_{0, j}\right)-\sum_{i=0}^{N} \mu_{i, 0}^{\top}\left(v_{i, 0}-\hat{v}_{i, 0}\right),
\end{aligned}
$$

the right hand-side of which is zero in view of (9-10).

Equations (4-10) constitute a two-dimensional two-point boundary value problem, and in general this is difficult to solve. On the other hand, in many cases of interest an explicit solution can be obtained. For example, when $R$ is positive 
definite, (7) can be solved for $u_{i, j}$, so that (6) and (8) can be written together as

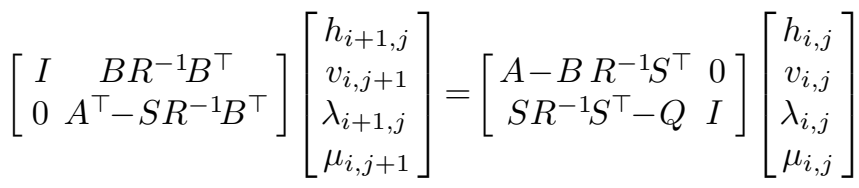

Note that the matrices appearing in the former are invertible if and only if $A-B R^{-1} S^{\top}$ is invertible. In this case, by using the general response formula given in [1], a matrix $\Phi^{i, j}$ can be explicitly determined yielding $\left[\begin{array}{llll}h_{i, j}^{\top} & v_{i, j}^{\top} & \lambda_{i, j}^{\top} & \mu_{i, j}^{\top}\end{array}\right]^{\top}=\Phi^{i, j} C$, where $C$ is a constant vector that can be computed by imposing the boundary conditions (4-5) and (9-10).

If $Q=S R^{-1} S^{\top}$, or in the even more particular case of minimum energy control as considered in [5, Section IV], where $Q$ and $S$ are both zero, the costate dynamic equation (6) can be solved backwards as described in [5], to obtain the same solution to this problem found for the first time in [7] by resorting to different techniques.

\section{A. Optimal cost}

For 1-D LQR optimal control problem, it is well-known that the optimal value of the performance index can be expressed as the difference $x^{\top}(0) \lambda(0)-x^{\top}(N) \lambda(N)$, where $x$ denotes the system state, $\lambda$ denotes the costate and $N$ is the length of the time horizon (i.e. frame of interest). In the following theorem it is shown that a similar result holds for 2-D Roesser models.

Theorem 2: Let $h, v, u, \lambda$ and $\mu$ satisfy equations (4-10). The optimal cost $J^{\star}$ is given by

$$
\begin{aligned}
J^{\star}= & \mathbf{h}_{N+1}^{\top} H \mathbf{h}_{N+1}+\mathbf{v}_{M+1}^{\top} V \mathbf{v}_{M+1} \\
& +\sum_{j=0}^{M}\left(h_{0, j}^{\top} \lambda_{0, j}-h_{N+1, j}^{\top} \lambda_{N+1, j}\right) \\
& +\sum_{i=0}^{N}\left(v_{i, 0}^{\top} \mu_{i, 0}-v_{i, M+1}^{\top} \mu_{i, M+1}\right)
\end{aligned}
$$

Proof: Let $x_{i, j} \triangleq\left[\begin{array}{ll}h_{i, j}^{\top} & v_{i, j}^{\top}\end{array}\right]^{\top}$ and

$$
c \triangleq \sum_{(i, j) \in \mathcal{Q}}\left[\begin{array}{ll}
x_{i, j}^{\top} & u_{i, j}^{\top}
\end{array}\right] \Pi\left[\begin{array}{l}
x_{i, j} \\
u_{i, j}
\end{array}\right] .
$$

By (7), (6) and (8) we get

$$
\begin{aligned}
c & =\sum_{(i, j) \in \mathcal{Q}} x_{i, j}^{\top} Q x_{i, j}+x_{i, j}^{\top} S u_{i, j}-u_{i, j}^{\top} B^{\top}\left[\begin{array}{l}
\lambda_{i+1, j} \\
\mu_{i, j+1}
\end{array}\right] \\
& =\sum_{(i, j) \in \mathcal{Q}} x_{i, j}^{\top}\left(\left[\begin{array}{l}
\lambda_{i, j} \\
\mu_{i, j}
\end{array}\right]-A^{\top}\left[\begin{array}{l}
\lambda_{i+1, j} \\
\mu_{i, j+1}
\end{array}\right]\right)-u_{i, j}^{\top} B^{\top}\left[\begin{array}{c}
\lambda_{i+1, j} \\
\mu_{i, j+1}
\end{array}\right] \\
& =\sum_{(i, j) \in \mathcal{Q}} x_{i, j}^{\top}\left[\begin{array}{l}
\lambda_{i, j} \\
\mu_{i, j}
\end{array}\right]-\left(A x_{i, j}+B u_{i, j}\right)^{\top}\left[\begin{array}{l}
\lambda_{i+1, j} \\
\mu_{i, j+1}
\end{array}\right] \\
& =\sum_{(i, j) \in \mathcal{Q}}\left[\begin{array}{ll}
h_{i, j}^{\top} & v_{i, j}^{\top}
\end{array}\right]\left[\begin{array}{l}
\lambda_{i, j} \\
\mu_{i, j}
\end{array}\right]-\left[\begin{array}{ll}
h_{i+1, j}^{\top} & v_{i, j+1}^{\top}
\end{array}\right]\left[\begin{array}{l}
\lambda_{i+1, j} \\
\mu_{i, j+1}
\end{array}\right]
\end{aligned}
$$

Since

$$
\begin{aligned}
\sum_{(i, j) \in \mathcal{Q}}\left(h_{i, j}^{\top} \lambda_{i, j}-h_{i+1, j}^{\top} \lambda_{i+1, j}\right) & \\
= & \sum_{j=0}^{M} h_{0, j}^{\top} \lambda_{0, j}-h_{N+1, j}^{\top} \lambda_{N+1, j}
\end{aligned}
$$

and

$$
\begin{aligned}
\sum_{(i, j) \in \mathcal{Q}}\left(v_{i, j}^{\top} \mu_{i, j}\right. & \left.-v_{i+1, j}^{\top} \mu_{i+1, j}\right) \\
& =\sum_{i=0}^{N} v_{i, 0}^{\top} \mu_{i, 0}-v_{i, M+1}^{\top} \mu_{i, M+1},
\end{aligned}
$$

the expression of the optimal cost readily follows.

\section{ACKNOWLEDGEMENTS}

This work was partially supported by the Australian Research Council (DP0664789).

\section{REFERENCES}

[1] R. Roesser, "A discrete state-space model for linear image processing," IEEE Transactions on Automatic Control, vol. AC-20, no. 1, pp. 1-10, 1975.

[2] E. Fornasini and G. Marchesini, "Doubly-Indexed Dynamical Systems: State-Space Models and Structural Properties," Mathematical System Theory, vol. 12, pp. 59-72, 1978.

[3] M. Bisiacco and E. Fornasini, "Optimal control of two-dimensional systems," SIAM Journal of Control and Optimization, vol. 28, pp. 582-601, 1990.

[4] M. Bisiacco, "New Results in 2D Optimal Control Theory," Multidimensional Systems and Signal Processing, vol. 6, pp. 189-222, 1995.

[5] C. Li and M. Fadali, "Optimal control of 2-D systems," IEEE Transactions on Automatic Control, vol. AC-36, no. 2, pp. 223-228, February 1991.

[6] O. Mangasarian, "Sufficient conditions for the optimal control of nonlinear systems," SIAM Journal on Control, vol. 4, no. 1, pp. 139$152,1966$.

[7] T. Kaczorek and J. Klamka, "Minimum energy control of 2-D linear systems with variable coefficients," International Journal of Control, vol. 44, no. 3, pp. 645-650, 1986. 